Nervenarzt 2022 · 93:512-519

https://doi.org/10.1007/s00115-021-01108-x

Angenommen: 22. Februar 2021

Online publiziert: 25. März 2021

(c) Der/die Autor(en) 2021

Mathilde Friederike Karoline Spiess wurde 1877 als viertes von acht Kindern des früheren Vikars und späteren Gymnasialprofessors Dr. phil. Bernhard Spiess und seiner Frau Johanna in Wiesbaden geboren [4, 40, 47, 49]. Fünf Kinder überlebten und Geld war knapp. Mit 16 Jahren beendete Mathilde die Ausbildung an der höheren Töchterschule und absolvierte 1895 das Lehrerinnenseminar. Nach einer Tätigkeit als Privatlehrerin wechselte sie an ein Mädchenpensionat in Biebrich. Ab 1900 - bis dahin war es für junge Frauen in Deutschland nicht möglich, das Abitur zu absolvieren - studierte sie mit einigem Einsatz und Ehrgeiz am ersten deutschen Mädchengymnasium in Karlsruhe, um in kurzer und damit wirtschaftlich vertretbarer Zeit das Abitur abzulegen, was ihr bereits im Juli 1901 mit nur drei weiteren Schülerinnen gelang. Mit 24 Jahren begann sie im Wintersemester 1901/02 in Freiburg im Breisgau wiederum mit nur drei weiteren Kommilitoninnen das Medizinstudium. Seit einigen Jahren war die Option „weiblicher Ärzte“ in Deutschland diskutiert worden, aber bisher praktizierten nur Ärztinnen, die im Ausland ausgebildet waren $[2,11,26]$. Großen Eindruck machten auf Mathilde die Vorlesungen des berühmten Zoologen August Weismann über die Deszendenztheorie. Das teure Studium finanzierte sie mit eigenem Erspartem, Privatunterricht, Vortragstätigkeit und mit Unterstützung des Allgemeinen Deutschen Frauenvereins. 1902 verlobte sie sich mit dem 4 Jahre jüngeren Gustav Adolf von Kemnitz, einem Kaufmannssohn aus gutem Hause, der nun auch den Plan entwickelte, zu studieren. Nach dem Physikum wechselte

Hans Förstl

Klinik und Poliklinik für Psychiatrie und Psychotherapie, TU München, München, Deutschland

\title{
Mathilde Ludendorff (1877-1966): Nervenärztin und völkische Philosophin
}

sie 1904 als Gasthörerin nach Berlin, wo sich Frauen noch nicht regulär immatrikulieren konnten, und heiratete von Kemnitz. Das junge Ehepaar zog 1905 nach München und beschäftigte sich mit den Ideen der Lebensreformbewegung und mit Lichtbildkunst (dabei entstandene Aktfotografien wurden später von missgünstigen Nationalsozialisten gegen sie verwendet). 1905 kam ihre Tochter Ingeborg$^{1}$ zur Welt, 1909 Zwillinge, die auf die Namen Asko und Hanno hörten.

\section{Emil Kraepelin und seine Lieblingsschülerin}

Im Wintersemester 1910/11 nahm sie das Medizinstudium wieder auf und hörte auch die Psychiatrievorlesung, in der sie bereits entscheidende Anregungen für ihr künftiges Werk erhielt: „Wer das Kolleg des Direktors der Psychiatrischen Klinik, Kraepelin, mit Aufmerksamkeit verfolgte und sich für das Gebiet besonders interessierte wie ich, der wusste ganz genau Bescheid, denn er führte uns viele Fälle des induzierten Irreseins vor, sprach auch über die Angstneurosen durch Höllenvorstellungen und die Entstehung des religiösen Wahnes ...“ [29, S. 214]. 1911 legte ihr Ehemann nach dem Studium

\footnotetext{
1 Mutter des Chemikers Walter E. Karg von Bebenburg (1927-1980), Inhaber mehrerer Patente, u. a. für das Schmerzmittel Flupirtin (Katadolon $\left.{ }^{(\mathrm{R})}\right)$; als Künstler: Walter E. Richartz, Übersetzer und von der Literaturkritik hochgelobter Autor [1], z. B. von „Tod den Ärtzten" [41] das u.a. eine ausführliche und ironische Auseinandersetzung mit Verschwörungstheorien enthält. Der Enkel spielt in den Erziehungswerken seiner Großmutter eine bedeutende Rolle.
}

der Zoologie und Physiologie bereits seine Dissertation vor (summa cum laude). Mathilde von Kemnitz bestand im April 1912 nach dem 10. Semester das Staatsexamen und übernahm bis Anfang 1913 eine Tätigkeit als Medizinalpraktikantin bei Professor Joseph Albert Amann in der II. Gynäkologischen Klinik (Standort Rotkreuz-Krankenhaus). Dort fertigte sie innerhalb von 6 Wochen ihre Dissertation an: Der asthenische Infantilismus des Weibes in seinen Beziehungen zur Fortpflanzungstätigkeit und geistigen Betätigung [20]. Darin argumentierte sie, eine "herabgesetzte Gebärtauglichkeit“ der Frauen und eine schlechte Gesundheit der Kinder seien nicht Folge geistiger, schöpferischer Anstrengung, sondern - falls vorhanden - in erster Linie angeboren; das sollte heißen, dass geistige Anstrengung Frauen nicht schadet. Wesentlicher Ansporn für ihre Auffassung war der Protest gegen eine „rassefremde Machtverteilung “ zwischen Mann und Frau, die unter anderem durch die fünf jesuitischen „Ks" verursacht sei: Kammer, Küche, Kirche, Kinder, Kleider [28, 46, S. 233-235]. Die Arbeit wurde im Mai 1913 erfolgreich verteidigt und danach in Ploetz' Archiv für Rassen- und Gesellschaftsbiologie veröffentlicht [20]. Im Juli 1913 erhielt sie die Approbation und wirkte danach als Volontärassistentin bei Professor Kraepelin: „Das Praktizieren bei dem berühmten Psychiater Kraepelin hatte mir manch anerkennendes Kopfnicken, ja auch Lob des wortkargen Mannes eingetragen, und er hatte sogar nach der Prüfung im Ärzteexamen gefragt, ob ich mich dem Gebiete der Psychiatrie zuwenden werde“ [25, S. 220]. Kraepelin war dem Einsatz 

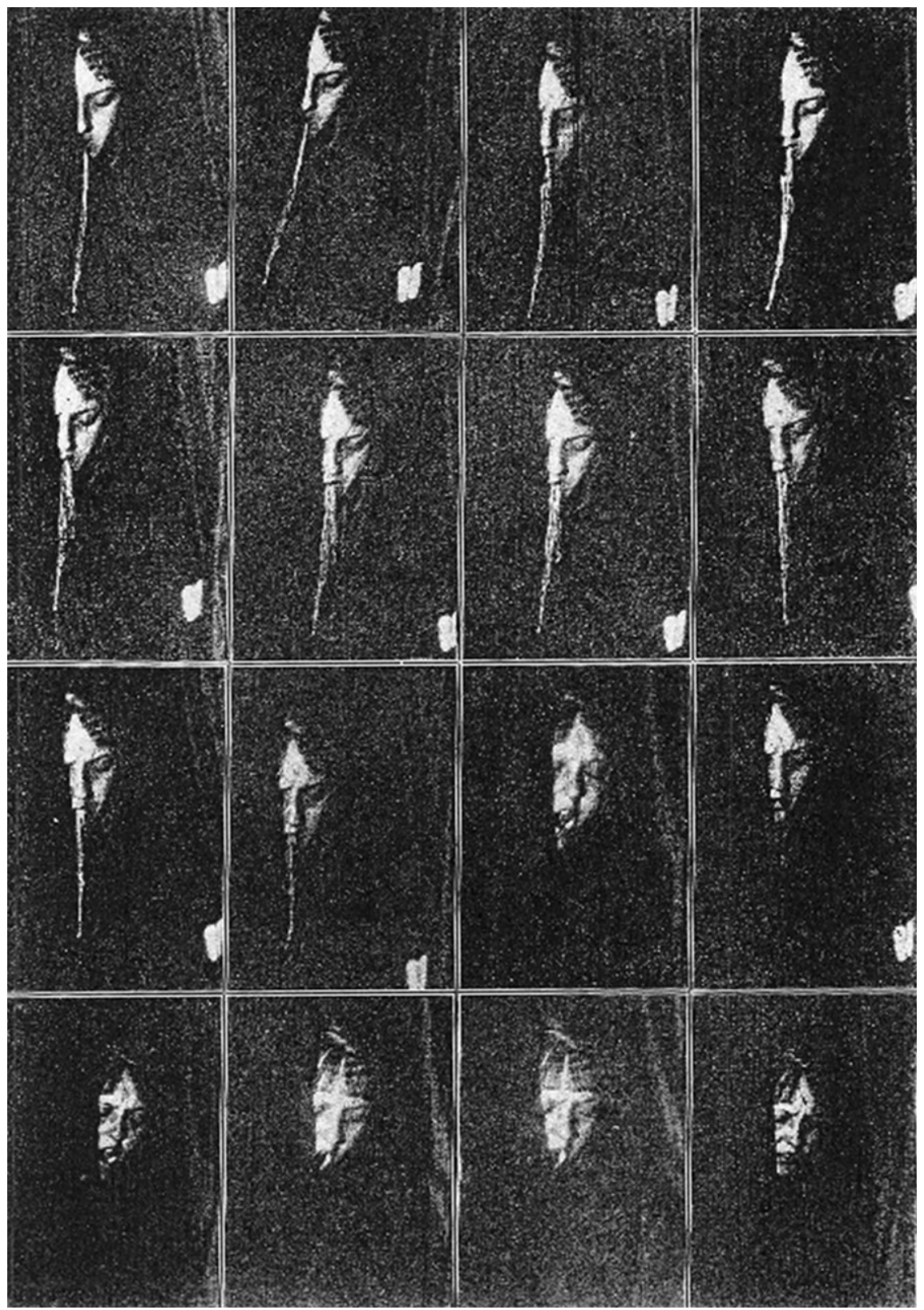

Abb. 1 ॥ Ausgewählte Bilder aus dem Film der kinematographischen Aufnahme am 13.07.1913. Breiter- und Schmalerwerden sowie Zurücktreten der Schleiersubstanz in den Mund (Tafel XXX; SchrenckNotzing, 1914 [43])

der ersten Ärztinnen - auch seiner eigenen Tochter Toni Kraepelin [39] - gegenüber aufgeschlossen, unter anderem da „vielfach das Erscheinen des Mannes auf der Frauenabteilung stark erregend wirkt (...) Da man anderwärts mit dieser Einrichtung gute Erfahrungen gemacht hat, wird sie sich voraussichtlich auch bei uns einbürgern, sobald einmal brauchbare Kräfte zur Verfügung stehen" [25]. Nach ihren Angaben war die persönliche Wertschätzung gegenseitig: „Dazu kam die geistige Anregung durch Professor ungeheuerlichen Zuständen das Weiterpflegen unheilbar zerstörter Menschen führt" [17, S. 230].

\section{Albert Freiherr von Schrenck- Notzing und die Medium- forschung}

In dieser Zeit gewann sie auch an Ansehen durch ihre kritische Auseinandersetzung mit der parapsychologischen Forschung des Dr. von Schrenck-Notzing, praktischer Arzt. Der lud die Münchner Gesellschaft zu Séancen in sein Palais und suchte Anerkennung in Kreisen der Wissenschaft $[8,45]$. Kraepelin betrachtete die obskure Mediumforschung, bei der es sich um meist oral ektoplasmatische Emanationen handelte, mit erheblicher Skepsis. Mathilde von Kemnitz gab Interesse vor und durfte an einer Sitzung teilnehmen. Dabei muss es sich um die Séanceam 13.07.1913 gehandelt haben, für die es recht abweichende schriftliche Berichte aus zwei Quellen gibt - von SchrenckNotzing [43] und von Kemnitz [21] sowie erstmals eine kinematographische Aufzeichnung (• Abb. 1). Schrenck-Notzing berichtete, das Medium gemeinsam mit einem Kollegen - „Dr. C. (Arzt)“ vor- und nachkontrolliert zu haben: „In diesen Sitzungen kam das bereits vorbekannte Schleierphänomen aus dem Munde zustande. Öffnung des Vorhanges erfolgte erst, nachdem die Materie produziert worden war" [43, Ss. 467-468].

Mathilde von Kemnitz hatte vorher schon versucht, das polnische Medium körperlich zu untersuchen was misslang: „Medium:lächelt, schüttelt den Kopf und sagt: ich nicht verstehe" [21, S. $50 \mathrm{ff}$.$] .$ Sie beschreibt auch, dass die Untersuchungen seitens der anderen Kollegen keineswegs lege artis durchgeführt werden konnte („sie ist kitzelig“). Zum parapsychologischen Phänomen selbst äußerte sich von Kemnitz höchst abfällig: „um 1 Uhr nachts die langwierige Herrlichkeit des Heraufwürgens einiger Lappen ..." $[29$, S. 236] und drohte sofort den Schwindel zu entlarven [29, S. 239]. Als Schrenck-Notzing eilends sein großes und reich bebildertes Werk über die Erforschung der mediumistischen Teleplastie veröffentlichte [43], war sie mit einer kritischen Replik schnell zur Stelle [21]. 
Ihre Arbeit erfuhr sogar eine wohlwollende Besprechung von Eugen Bleuler ${ }^{2}$ [3] und führte zu einer Vortragseinladung auf dem Straßburger Jahreskongress des Deutschen Vereins für Psychiatrie im April 1914, einer Veranstaltung, bei der diesmal die Parapsychologie aufs Korn genommen wurde, nachdem im Jahr zuvor die Psychoanalyse zerlegt worden war [9]. Für Schrenck-Notzing war Mathilde von Kemnitz eine Katastrophe, „imstande den wissenschaftlichen Fortschritt auf Jahre hinaus zu hemmen “ [8, $44,45]$. Für Mathilde von Kemnitz bedeutete der Erfolg eine weitere Motivation für den Kampf gegen obskure Lehren, induziertes Irresein und Verschwörungen aller Art. Sie erweiterte die Front nachdem sie ihren Verstand an Kant, Nietzsche und Schopenhauer geschärft hatte.

\section{Sigmund Freud und die Seelenkunde}

Durchsetzungswille prägte diese Lebensjahre auf ganzer Linie. Gustav Adolf von Kemnitz hatte 1914 seine Habilitationsarbeit vollendet (Beiträge zur Kenntnis des Spermatozoen-Dimorphismus), jedoch „unserer Ehe heilige Ausschliesslichkeit hatte nicht gewaltet" [29, S. 208]. Bei Mathilde wurde eine Lungentuberkulose diagnostiziert und sie begab sich nach Bozen zur Kur, wo sie an ihrem Buch Das Weib und seine Bestimmung zu arbeiten begann. Privatdozent Gustav Adolf wurde wegen Kurzsichtigkeit mehrfach für wehruntauglich befunden, die Finanzen waren durch Fehlspekulationen verloren und sie war nach verhältnismäßig kurzer Weiterbildungszeit ${ }^{3}$ gezwungen, eine Anstellung als Spezialärztin für Nervenheilkunde in einem Kur- und Offiziersgenesungsheim in Garmisch anzunehmen, wo sie sich dann beruflich selbständig machte. In dieser Zeit entstand eine Arbeit über traumatische Kriegsneurosen bei Offizieren [22]. Ihr

\footnotetext{
2 Dies ist nicht nur als Zeichen der Anerkennung in der Fachwelt erwähnenswert, sondern vor allem wegen Bleulers eigenem Interesse am Okkultismus [8].

3 Richtlinien zu Inhalt und Dauer der Facharztweiterbildungen wurden erst 1924 beschlossen [19].
}

Nervenarzt 2022 -93:512-519 https://doi.org/10.1007/s00115-021-01108-x

(c) Der/die Autor(en) 2021

\section{H. Förstl}

\section{Mathilde Ludendorff (1877-1966): Nervenärztin und völkische Philosophin}

\section{Zusammenfassung}

Mathilde Ludendorff (geb. Spiess, verw. von Kemnitz, gesch. Kleine) war eine der ersten Frauen, die im wilhelminischen Deutschland Medizin studierte. Sie schrieb eine feministische Dissertation; gab an, sehr früh Sigmund Freuds Psychoanalyse erfolgreich widerlegt zu haben und zu Emil Kraepelins bester Schülerin avanciert zu sein; deckte den Schwindel von Albert Schrenck-Notzings Mediumforschung auf; firmierte nach 17-monatiger Weiterbildung als Spezialärztin für Nervenheilkunde; behandelte General von Ludendorffs erste Frau und wurde alsbald seine zweite; entwickelte eine germanische Philosophie, die Adolf Hitler zu verwegen erschien; wurde
1949 in einem Spruchkammerverfahren zunächst als Hauptschuldige verurteilt und setzte sich kritisch mit dem Gutachten ihres Kollegen Georg Stertz über ihren eigenen Geisteszustand auseinander. Ihre zahlreichen Schriften werden weiter verlegt. Der von ihr begründete „Bund für Gotterkenntnis (Ludendorff)" existiert noch und wird vom Verfassungsschutz beobachtet.

Schlüsselwörter

Sigmund Freud - Geschichte der Nervenheilkunde - Emil Kraepelin · Erich Ludendorff . Nationalsozialismus - Albert von SchrenckNotzing · Georg Stertz

\section{Mathilde Ludendorff (1877-1966): specialist for nervous and mental diseases and Germanic philosopher}

\begin{abstract}
Mathilde Ludendorff (nee Spiess, widowed von Kemnitz, divorced Kleine) was one of the first women who studied medicine in Imperial Germany. She wrote a feminist doctoral thesis, refuted Sigmund Freud's psychoanalysis early in her career, detected the fraud of Albert von Schrenck-Notzing's spiritualist research, became a specialist for nervous and mental diseases after only 17 months of training with Emil Kraepelin, as his-according to her own words-best pupil, treated General Ludendorff's first wife and soon became his second, developed a Germanic philosophy too radical for Adolf Hitler's taste, was
\end{abstract}

considered as a primary culprit after a first denazification trial in 1949 and contested the expert opinion of her colleague Professor Georg Stertz about her own mental state. Her books are still in print and her Alliance for God Cognizance (Ludendorff) still exists and is monitored by the National Intelligence Agency.

\section{Keywords}

Sigmund Freud - History of neuropsychiatry . Emil Kraepelin · Erich Ludendorff · National Socialism · Albert von Schrenck-Notzing · Georg Stertz
Mann nahm mit seiner jüdischen Geliebten eine Wohnung in Schwabing. Zum Jahreswechsel 1916/17 wurde er auf einer Skitour mit zwei Begleiterinnen von einer Lawine verschüttet, sein dritter Bergunfall in kurzer Folge und diesmal tödlich [38, 47].

Mathilde von Kemnitz hatte nach eigenen Worten „die Freud'sche Theorie... schon in meinen Assistentenjahren widerlegt, ohne dass meine Abhandlung in die Fachpresse aufgenommen worden wäre“ [29, S. 273]. ... „Die sogenannte Freudsche, Psychoanalyse' hat die Gesetze des Unterbewusstseins zu gerin- gerem Teil begriffen, aber in ihren Ursachen und Zusammenhängen so traurig missverstanden ... “ $[28$, S. 160]. ... „Die Lehre der Psychoanalyse Freuds, dies verdrängte Erlebnis sei stets ein sexuelles, ist ein verheerender Irrtum, der sich aus seiner jüdischen Rasseeigenart etwas erklären und entschuldigen lässt" [28, S. 162]. ... „Die Art, wie er (Freud) die Sexualität überhaupt anfasst, die Art wie er sie durch Zotenumdeutungen $\mathrm{zu}$ heilen versucht, indem er die ,freien Assoziationen' und die Träume im Sinne von Zoten auslegt, ist ein volksverseuchender Irrsinn. Er wird nur begreiflich, 
Tab. 1 Auswahl von Mathilde Ludendorffs umfangreicheren Schriften

1913 Der asthenische Infantilismus des Weibes in seinen Beziehungen zur Fortpflanzungstätigkeit und geistigen Betätigung (Med. Diss.)

1914 Ein Blick in die Dunkelkammer der Geisterseher: moderne Medium-„Forschung“: kritische Betrachtung zu Dr. von Schrenck-Notzings "Materialisationsphaenomene“

1917 Das Weib und seine Bestimmung. Ein Beitrag zur Psychologie der Frau und zur Neuorientierung ihrer Pflichten

1919 Erotische Wiedergeburt (später: Der Minne Genesung)

1920 Des Weibes Kulturtat

1921 Triumph des Unsterblichkeitswillens

1923 Der Seele Ursprung und Wesen (3 Bände)

I. Schöpfungsgeschichte

1925 II. Des Menschen Seele

1927 III. Selbstschöpfung

1927 Deutscher Gottglaube

1929 Das Geheimnis der Jesuitenmacht und ihr Ende (mit E. Ludendorff)

1930 Der Seele Wirken und Gestalten

I. des Kindes Seele und der Eltern Amt

1933 II. die Volksseele und ihre Machtgestalter

1935 III. das Gottlied der Völker - eine Philosophie der Kultur

1933 Induziertes Irresein durch Occultlehren

1934 Christliche Grausamkeit an Deutschen Frauen (mit Walter Löhde)

1936-68 Statt Heiligenschein oder Hexenzeichen - mein Leben, 6 Bände

1939 Die Judenmacht, ihr Wesen und Ende

1941 Der Siegeszug der Physik - ein Triumph der Gotterkenntnis meiner Werke

1950 Wunder der Biologie im Lichte der Gotterkenntnis meiner Werke

1957 Das Hohe Lied der göttlichen Wahlkraft

1959 In den Gefilden der Gottoffenbarung

1960 Das Jenseitsgut der Menschenseele

I. Der Mensch, das grosse Wagnis der Schöpfung

$1961 \quad$ Unnahbarkeit des Vollendeten

1962 wenn wir sehen, wie völlig die Phantasie dieses chronisch überreizten Juden und seine Heilverfahren mit bestimmten Teilen des Talmuds übereinstimmen. Sein Erbgut im Unterbewusstsein ermöglicht diese Auffassung von der Seele und ihren Gesetzen.... Wer die genannten Gesetze erkannt hat, der wird in der leider eingeführten ungeheuer unbeholfenen und umständlichen, mechanischen Weise ein Unvermögen sehen, das verdrängte Erlebnis durch ,freie Assoziationen' zu finden" [28, S. 165-166]. Sie selbst habe nur bei $5 \%$ aller Neurosen „sexuelle Erlebnisse" gefunden. Mit diesen Darlegungen werden Kraepelins Gedanken aus der 8. Auflage seines Lehrbuches drastisch paraphrasiert. Er fand die Auffassungen Freuds höchst eigentümlich: „Jedenfalls genügen die Aussagen einer Reihe auf das stärkste suggestiv beeinflussbarer Personen nicht entfernt, um ken in einer Reihe weiterer Schriften dargelegt (• Tab. 1; • Abb. 2). Im August kam es nach einer Parteikundgebung in der Murnauer Turnhalle zu einer Begegnung mit Adolf Hitler in privatem Rahmen [14, S. 110]: „Frau von Kemnitz widerfuhr bei dieser Gelegenheit das Missgeschick, mit ihrer, von einem durchsichtigen Chiffonkleid eingehüllten imposanten Körpersilhouette derart in das Hintergrundlicht dieser weissen Nacht zu geraten, dass eine stehend von ihr erteilte Vorlesung über Rasseaufzucht und Freikörperkultur gleich eine recht anschauliche plastische Untermalung erfuhr. ... An jenem Abend gestaltete sie ihre Auslassungen über Weltgeist und Weltall ein wenig zu ermüdend; schließlich wandte sie sich mit ihrer Forderung nach einer neuen, im nordischen Ahnenerbe wurzelnden Religion direkt an Hitler." Hitler reagierte abwehrend, er wolle nicht ausschließen, dass es möglicherweise irgendwann einmal einen Philosophen geben werde, der, aus dem was wir wollen, ein Glaubenssystem entwickeln werde. „Darauf Frau Mathilde, sich $\mathrm{zu}$ voller Körpergrösse straffend und das Denkerhaupt empor gereckt als lausche sie bereits dem Flügelschlag der Ewigkeit: Dieser Philosoph, Herr Hitler, steht bereits vor Ihnen!"Er verabschiedete sich daraufhin umgehend und wortlos [14, S. 111]. Noch 1942 äußerte sich Hitler im Tischgespräch unvorteilhaft über früher gewonnene Eindrücke [17]: „1924 tauchten bei mir die politischen Weiber auf: die Frau [Ehrengard] von Treuenfels, die Mathilde von Kemnitz, sie wollten Reichstagsmitglieder werden, um die Sitten dort zu veredeln. Ich sagte Ihnen, neunundneunzig Prozent aller Beratungsgegenstände sind Männerdinge, die sie nicht beurteilen können!“

Ab 1925 behandelte sie die morphiumsüchtige Ehefrau Erich Ludendorffs, Margarethe, von der er sich dann im Juli 1926 scheiden ließ, um im September des gleichen Jahres Mathilde auf dem Standesamt in Tutzing zu ehelichen. Dennoch fand die geschiedene Margarethe in ihren etwas konfusen Erinnerungen („bei dem Hitler-Putsch passierte eine amüsante Geschichte ... “; [32, S. 309]) fast nur gute Worte für ihren geschiedenen Gatten, außer „Menschenkennt-
Im Jahr 1919 heiratete Mathilde von Kemnitz Major a. D. Edmund G. Kleine. Die Ehe wurde 1922 geschieden. Bis 1923 hatte sie ihre weltanschaulichen Gedan- 


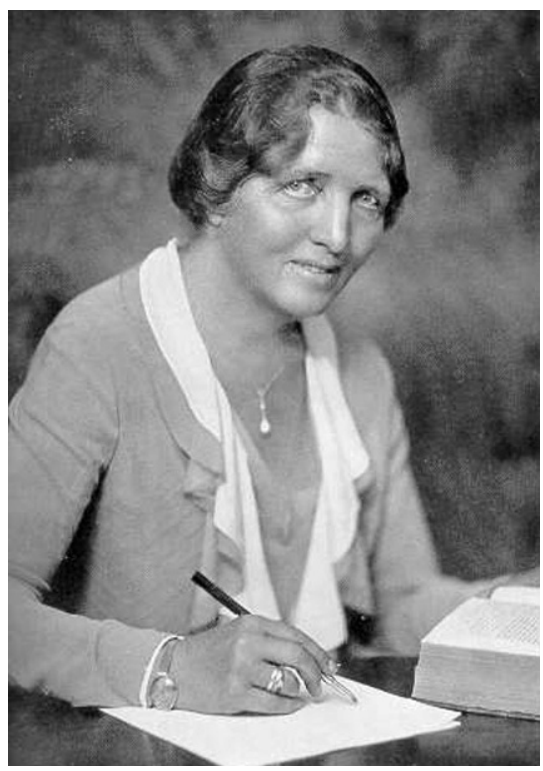

Abb. $2 \Delta$ Mathilde von Ludendorff als Autorin. (Inv.Nr. A57/4508<2> @) Deutsches Historisches Museum, Berlin)

nis hat Ludendorff nie besessen, sonst könnte er nicht immer Einflüssen unterliegen, die ihn ins Unglück stürzen“ [32, S. 276]. Mathilde Ludendorff schildert im Kapitel Der Menschen Niedertracht öffnet dem Glück die Tore im Band 5 ihrer Autobiographie Freiheitskampf wider eine Welt von Feinden [31] wie sie versuchte, mit ihrem ärztlichen Gewissen ins Reine zu kommen: „Wie schwer und unerquicklich ist doch der Arztberuf. Hätte die Patientin die Widerstandskraft bewahrt, dann war ich der Engel, der sie gerettet hatte. Nun trafen mich mit einem Mal Hass und Verleumdung. Gut, dass Ludendorff das nicht ahnte" [32, S. 47]. Es werde behauptet „eine Ärztin, Frau Dr. v. Kemnitz, habe unter dem Deckmantel ärztlicher Hilfe die Ehe unterwühlt, und Ludendorff sei ihrer Taktik erlegen. So etwa lauteten diese Mitteilungen, die in geringen Abwandlungen in den verschiedensten Zeitungen in ganz Deutschland auftauchten und meine Frauen- und Berufsehre besudeln sollten“ [32, S. 49]. ... „Und sieh da, der Tag war gekommen, der mich in eine Welt des Glückes stellte, eine so völlig andere Welt der seelischen Erfüllung in Zweisamkeit. Es hatte der einsame, für alles seelisch Unnahbare, Verschlossene, Grosse, der sich ein Leben lang - oft sogar von seiner nächsten Umgebung -

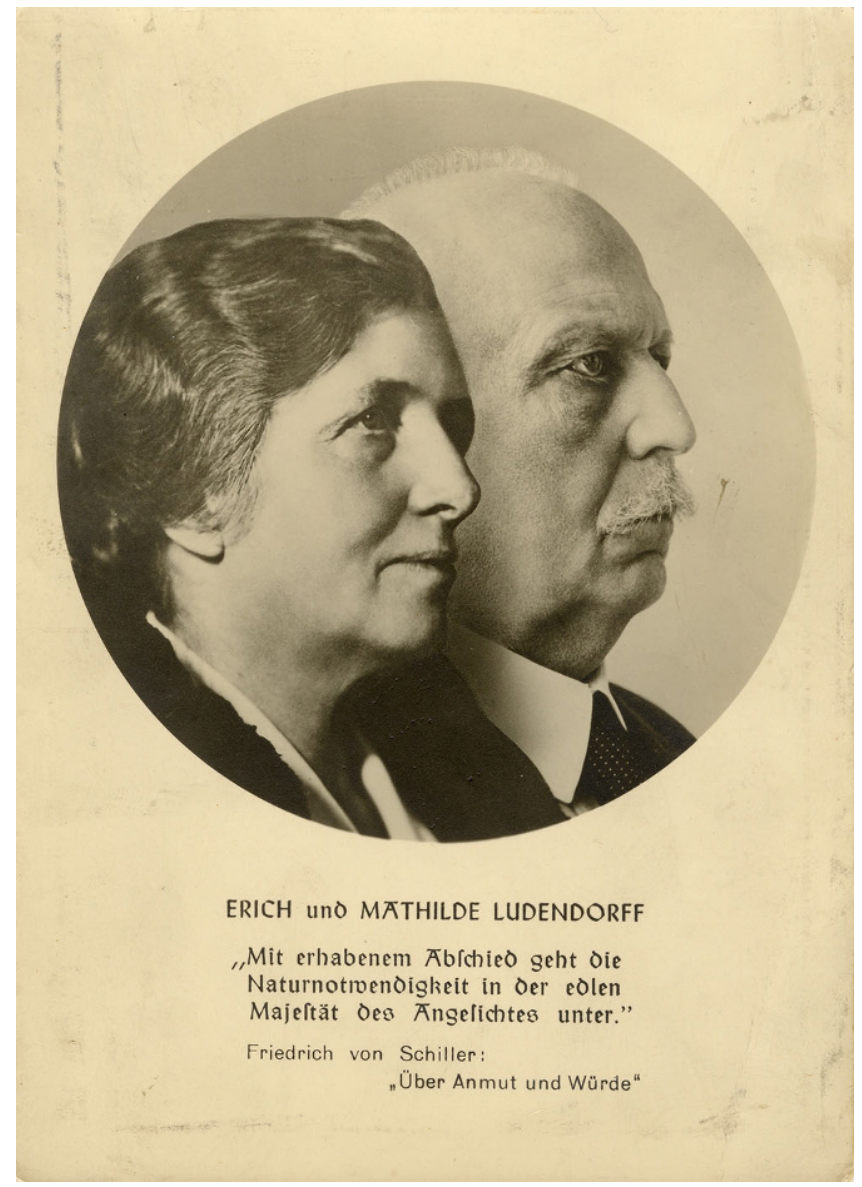

Abb. $3<$ Doppelportrait Mathilde und Erich Ludendorffzum Gedenken an den 1. Jahrestag des Todes von Erich Ludendorff, 20.12.1938. Das schwer verständliche Zitat stammt tatsächlich von Friedrich Schiller [42], der nach fester Überzeugung der beiden von Freimaurern ermordet worden war, wie übrigens auch Lessing und Mozart (Sammlung Dr. Ulrich Prehn, Berlin)

lieber missverstehen liess, als Unebenbürtigen eine Wirkung auf sein Seeleninneres zu gewähren, seine Seele aufgetan“ [32, S. 53]. Mit Erich Ludendorff (1865 bis 1937) heiratete sie die Personifikation des deutschen Kriegshelden und seiner Schmach, den deutschen De-factoStrategen des 1. Weltkriegs, der 9 Mio. tote Soldaten mitzuverantworten hatte, sich zur Ehrenrettung der Dolchstoßlegende bediente, gerade bei der Wahl zum Reichspräsidenten mit nur 300.000 Stimmen abgestraft worden war und später unter Mathildes Einfluss den totalen Krieg als geballte Anstrengung der „seelischen Entschlossenheit" einer ganzen Nation ersann [27].

Hier begegneten sich verwandte Seelen: „Das nordische Gotterleben, also vor allem gekennzeichnet durch das klar bewusste Erleben des Gottes in der eigenen Seele, vererbt hiermit die starke Entfaltung des Gottesstolzes im Rassecharakter. Daher der Freiheitswille und das Herrenbewusstsein des Germanen“ [30, S. 137]. Ludendorffs kongeniale Einschätzung seiner Gattin [7]: „Sie zeigte Wirken der Volksseele das heisst des Rasseerbgutes im Unterbewusstsein - wie es Gott erlebt und dem Göttlichen gegenübersteht mit den Charaktereigenschaften der Rasse - in den einzelnen Volkskindern und wie sie in rassereinen Völkern, aber selbst noch in rassegemischten, deren Hüterin werden kann." Zwei verwandte Seelen hatten sich gefunden, gründeten 1928/29 Ludendorffs Volkswarte Verlag, 1937 - mit zögerlicher Erlaubnis Hitlers, der Ludendorff wieder für sich gewinnen wollte - den völkisch-religiösen Bund für Deutsche Gotterkenntnis (L) [L für Ludendorff] und feierten einander in hohem Ton (• Abb. 3), manövrierten sich damit jedoch in eine immer extremere Außenseiterposition. Goebbels [13]: „Der Chef spricht sich scharf gegen Ludendorff, vor allem gegen seine Frau aus" (29.05.1929). „Es ist schade um den grossen General. Seine Frau ist ein böser Geist" (23.05.1937). Erich Ludendorff starb im Dezember 1937 
in München. $\mathrm{Zu}$ Kriegsbeginn wurde dem Ludendorff-Verlag das zugestandene Papierkontingent prompt entzogen, sodass auch die Halbmonatsschrift Am Heiligen Quell Deutscher Kraft versiegte. Mathilde und Erich Ludendorff hatten sich in ihre eigene ultravölkische Lehre hineingesteigert und den Nationalsozialismus Hitlers sichtlich geringgeschätzt. Sie waren keine Mitglieder der NSDAP oder anderer nationalsozialistischer Organisationen. Dennoch stand Mathilde im Verdacht, intellektuelle (Mit-)Urheberin des NS-Gewaltstaates zu sein, und musste sich - wie sehr viele andere im Rahmen der Entnazifizierung einem Spruchkammerverfahren stellen $[6,47]$.

\section{Georg Stertz und das Spruchkammerverfahren}

Georg Stertz (1878-1959) war 1937 wegen seiner Ehe mit der "halbjüdischen“ Tochter Aloys Alzheimers in Kiel als Direktor der Psychiatrischen Universitätsklinik zwangsemeritiert worden und gelangte 1947 zu der späten Ehre, Bumkes Nachfolger an der Münchner Nervenklinik zu werden. Im gleichen Jahr ereilte ihn der Auftrag von der Spruchkammer des Landkreises Starnberg, ein Gutachten über den Geisteszustand Mathilde Ludendorffs zu verfassen. Einer Bekannten Mathilde Ludendorffs gegenüber äußerte Stertz im Mai 1947, „dass es besser wäre, man würde Frau Dr. Ludendorff als nicht ganz geistig verantwortlich für ihre Werke erklären. Dann wäre das Verfahren schnell beendet. Diese Frau Dr. von Unruh wies dies Ansinnen mit der Erwiderung zurück ... dass sie völlig gesund sei und von Prof. Kraepelin seinerzeit als seine beste Schülerin bezeichnet worden sei ..." [50].

Das Gutachten mit Datum vom 22.09.1947 [48] nimmt zu den Fragen Stellung, ob Mathilde Ludendorff für ihr Tun und für ihre Werke in Vergangenheit und Gegenwart die volle Verantwortung besitze (damals $₫ 51 \mathrm{StGB}$ ) und ob sie einweisungsfähig für ein Arbeitslager sei. Die zweite Frage wollte Stertz angesichts der „annähernd 50-jährigen“ (sie war damals fast 70) Patientin nicht beantworten. Auf S. zwei steht „nach Bekundungen einiger verlässlicher Zeu- gen soll (Mathilde Ludendorff) ... zu Beginn ihrer ärztlichen Tätigkeit einen auffälligen, extravaganten Eindruck gemacht haben, der sich in ihrem Äusseren und in ihrem Benehmen zu erkennen gab. "Kurz erwähnt werden Studium und ärztliche Tätigkeit sowie die Hinwendung zur schriftstellerischen Tätigkeit nach der Lektüre Kants und Schopenhauers mit dem propagandistischen Ziel einer Bekämpfung überstaatlicher Mächte. $\mathrm{Ab}$ der fünften Seite mischen sich Stertz' grundsätzliche psychopathologische Überlegungen und diagnostische Einschätzungen über das Ehepaar Ludendorff, insbesondere hinsichtlich der vollkommenen Arbeitsgemeinschaft und der Grenzen von Selbstüberschätzung und Größenwahn. Auf S. 16 gelangt Stertz zu dem Schluss das ,gesamte Verhalten von Frau Dr. Ludendorff, ihre Intelligenz, ihre lebhafte jeder Anregung zugewandte Auffassungsgabe, ihr Temperament, ihr ungestörtes Gedächtnis, das alles spreche gegen das Bestehen einer geistigen Erkrankung. ..." Es gebe keinerlei Anhaltspunkte dafür, dass sie nicht voll verantwortlich wäre.

Mathilde Ludendorff, Facharzt für Psychiatrie [30], ließ dem Gericht eine 14-seitige Kritik des Gutachtens zukommen, in dem sie unter anderem die lange Latenz in der Gutachtenerstellung monierte, falsche Angaben hinsichtlich Zeit der Assistententätigkeit bei Kraepelin (nicht 1 Jahr, sondern 1,5, nämlich vom 01.08.1912 bis 01.01.1914). Das Gutachten sei vom Standpunkte des Facharztes aus „geradezu ungeheuerlich“, u. a. da es sich der Werke des Pamphletisten Martini [33] bediene $e^{4}$. Zur Selbstüberschätzung könne Stertz kein Urteil abgeben, da er keine Zeit gehabt habe, ihre Leistung, ihre Werke zu würdigen. Stertz' „psychiatrisches Kolleg“ über Wahn gehöre nicht in das Gutachten. „Er selbst hat sein Liedlein von den armen Juden gesungen." Nach ihrem Eindruck schien er keine genauen Aufzeichnungen über

\footnotetext{
${ }^{4}$ Der Journalist Winfried Martini war von Staatsanwalt Julius Herf ebenfalls zu einer Stellungnahme aufgefordert worden und hatte bei Ludendorffs die Diagnose eines "germanisch-depressiven Irreseins" gestellt [5, 33].
}

die Unterredungen gemacht $\mathrm{zu}$ haben, daher die Erinnerungsirrtümer.

Mathilde Ludendorff dagegen hatte selbst ein detailliertes 31-seitiges, teilweise wörtliches Gedächtnisprotokoll über die insgesamt fünf Begegnungen angefertigt [30]. Ende Mai habe er sie gefragt, ob sie okkult-gläubig sei, also gar nichts über ihre Arbeit gewusst. Anfang Juni sei er auf die psychische Erkrankung ihrer älteren Schwester zu sprechen gekommen um dann anzumerken ,und dann haben Sie sich auf einmal ... zugetraut, in das schwierige Problem der Atomphysik einzudringen? (diese Frage ist von besonders ironischem Lächeln begleitet).“ Mitte Juni habe er sich über die dichterische Qualität ihres Werkes mokiert: „er bricht in Lachen aus und kann zunächst gar nicht damit aufhören, unterbricht auch im Folgenden immer wieder sein Sprechen mit höhnischem Lächeln oder Lachen. Sie haben behauptet, dass die Freimaurer Schiller, Lessing, Mozart und andere ermordet hätten und das nenne ich Wahnsinn." Am 26.07.1947 um 16:30 findet die letzte Begegnung statt: „Professor Stertz empfängt mich in seinem Schlafzimmer, sitzt in Pyjama im Sessel, spricht nur sehr leise und matt. Wir bedauern gegenseitig das durchlebte Kranksein.... Die Frau Professor kommt und sagt, ihr Mann müsse nun ins Bett, die Stunde sei vorüber. Frau Professor will mir die Bücher zurückgeben, führt mich von der Privatwohnung durch die Männerabteilung der Klinik und führt mich in ein Zimmer, an dessen Tür das Schild: ,Dr. Röhrig' hängt. Dort liegen auf dem Schreibtisch alle meine Bücher, die sie mir mitgibt."

\section{Epilog}

Mathilde Ludendorff wurde im ersten Spruchkammerverfahren als Hauptschuldige verurteilt. 1951 wurde das Urteil revidiert und abgemildert und sie wurde als "Belastete“ eingestuft, die den Nationalsozialismus als Aktivistin unterstützt habe. Hauptschuldigen (Kategorie I) drohten ursprünglich 2 bis 10 Jahre, Belasteten (Kategorie II) bis zu 5 Jahre Arbeitslager mit nachfolgendem Arbeitsverbot und Enteignung. Auch Minderbelastete (III) und Mitläu- 
fer (IV) standen in der Gefahr, Arbeit, Ansprüche auf Lebensmittelmarken und Rente $\mathrm{zu}$ verlieren. Dabei gab es allerdings zwischen und auch innerhalb der Besatzungszonen keine einheitlichen Richtlinien, jedoch eine deutliche Abmilderung der Strafmaße innerhalb ganz weniger Jahre; nur 0,7\% der Beschuldigten wurden als Hauptschuldige oder Belastete eingestuft [5, 6, 36]. 1951 wurde der völkische „Bund für Gotterkenntnis" wieder ins Leben gerufen und 1961 als verfassungsfeindlich verboten. 1971 wurde der Bund wegen Verfahrensfehlern wieder zugelassen. Mathilde Ludendorff starb 1966 im Alter von 88 Jahren in Tutzing an einer Urämie [4, 40, 47, 49]. Die Bücher von Mathilde Ludendorff werden weiterhin verlegt (hohewarte-online.de). Der Verlag versucht sich auch lebhaft in aktuelle Debatten einzubringen. Die Ludendorff-Villa in Tutzing steht seit 2010 unter Denkmalschutz und den Verein für Gotterkenntnis (L) gibt es immer noch: „Wenn Sie Verbindung mit uns aufnehmen wollen, schreiben Sie uns an folgende Anschrift oder schicken Sie uns eine E-Post (E-Mail) über unser Kontaktformular: Weltanschauungsgemeinschaft Bund für Gotterkenntnis (Ludendorff) e. V., Postfach 1254, 82324 Tutzing/Oberbayern. E-Post: info(AT)ludendorff.info.“

Es gibt keine zuverlässigen Zahlen, wie viele dem Bund für Gotterkenntnis noch anhängen, aber während anhaltender Krisen und in Phasen der Orientierungslosigkeit nimmt das Interesse an Querdenken und alternativen Erklärungsmodellen der Realität zu. Sie bieten angesichts unübersichtlicher Verhältnisse schlüssige (konklusive), dank höheren Wissens exklusive und wegen der Gruppenzugehörigkeit inklusive Alternativen [10]. Während der Weimarer Republik erstarkten neu-heidnische Glaubensbewegungen, die alte Kulte reaktivierten, z. B. Wilhelm Hauers Deutsche Glaubensbewegung, Sigrid Hankes Unitarier und eben auch Ludendorffs Deutscher Gottglaube [23, 37, 47, 49]. Ein anderer Kraepelin-Schüler, Willi Hellpach, der 1925 deutlich erfolgreicher als Ludendorff für das Amt des deutschen Reichspräsidenten kandidiert hatte, profilierte sich auch als Pantheist, wirkte aber gleichzeitig als Realpolitiker, begründete die Umweltpsychologie und beförderte die Psychosomatik [15, 16]. Faszination und Faschismus hängen zusammen. Der Schwung des Neo-Paganismus wurde von den Ideologen Feser und Rosenberg für die Hitler-Bewegung aufgenommen - wobei sich Ludendorffs partout nicht fügen wollten [14, 23, 37, 49]. Hitlers gewählt ekstatische Rhetorik und seine inbrünstigen Posen schöpften aus dieser quasi-religiösen Quelle.

Das Wesen von Mathilde Ludendorffs schillernden Werken lässt sich nicht einfach ergründen. Es reicht von antiokkultistisch bis esoterisch, feministisch bis pantheistisch, antiklerikal bis antisemitisch, völkisch bis verschwörungstheoretisch und verdient das Interesse von Nervenärzten - auch hinsichtlich der Begutachtung narzisstischer und querulatorischer Personen mit Fachkenntnissen.

\section{Korrespondenzadresse}

\section{Prof. Dr. Hans Förstl}

Klinik und Poliklinik für Psychiatrie und

Psychotherapie, TU München

Ismaningerstr. 22, 81675 München,

Deutschland

hans.foerstl@tum.de

Danksagung. Dr. Christoph Bachmann, Staatsarchiv München; Barbara Hutzelmann, Stadtarchiv München; Dr. Judith Niedermaier, Bayerische Landesärztekammer; Dr. Ulrich Prehn, TU Berlin; Dr. Annika Spilker, Eschwege.

Funding. Open Access funding enabled and organized by Projekt DEAL.

Interessenkonflikt. H. Förstl gibt an, dass kein Interessenkonflikt besteht.

Open Access. Dieser Artikel wird unter der Creative Commons Namensnennung 4.0 International Lizenz veröffentlicht, welche die Nutzung, Vervielfältigung, Bearbeitung, Verbreitung und Wiedergabe in jeglichem Medium und Format erlaubt, sofern Sie den/die ursprünglichen Autor(en) und die Quelle ordnungsgemäß nennen, einen Link zur Creative Commons Lizenz beifügen und angeben, ob Änderungen vorgenommen wurden.

Die in diesem Artikel enthaltenen Bilder und sonstiges Drittmaterial unterliegen ebenfalls der genannten Creative Commons Lizenz, sofern sich aus der Abbildungslegende nichts anderes ergibt. Sofern das betreffende Material nicht unter der genannten Creative Commons Lizenz steht und die betreffende Handlung nicht nach gesetzlichen Vorschriften erlaubt ist, ist für die oben aufgeführten Weiterverwendungen des $\mathrm{Ma}$ terials die Einwilligung des jeweiligen Rechteinhabers einzuholen.
Weitere Details zur Lizenz entnehmen Sie bitte der Lizenzinformation auf http://creativecommons.org/ licenses/by/4.0/deed.de.

\section{Literatur}

1. Arzt G (1995) Walter E. Richartz - über literarische und naturwissenschaftliche Erkenntnis. Igel, Paderborn

2. Binder S(1892) WeiblicheÄrzte.Göschen, Stuttgart

3. Bleuler E (1914) Rezension von Kemnitz: Moderne Mediumforschung. Munch Med Wochenschr 61:429

4. Burgmair W, Engstrom EJ, Weber MM (2006) Kraepelin in München I, 1903-1914. Belleville, München, S34-37

5. Der Spiegel (1949) Am heiligen Quell Deutscher Kraft. 26:

6. Der Spiegel (1960) Mathilde Ludendorff Gotterkenntnis (L). 8:22-32

7. Der Spiegel (1968) Erster Weltkrieg - Krümel in der Hand. Der Spiegel, 12:52

8. Dierks M (2012) Thomas Manns Geisterbaron. Leben und Werk des Freiherrn Albert von Schrenck-Notzing. Psychosozial-Verlag, Gießen

9. Falzeder EM, Burnham JC (2007) A perfectly staged concerted action against psychoanalysis: the 1913 Congress of German psychiatrists. Int J Psychoanal 88:1223-1244

10. Förstl H (2020) Die COVID-19 Verschwörung in Theorie und Praxis. Dtsch Med Wochenschr 145:1870-1875

11. Förstl H (2020) Emma Wilson Mooers (1858-1911): die Neuropathologin an Aloys Alzheimers Seite. Nervenarzt 91:1146-1148

12. Förstl H (2021) Katatones Wandern mit Emil Kraepelin, Aloys Alzheimer, Henry Aloysius Cotton et al. Nervenarzt. https://doi.org/10.1007/s00115020-01039-z

13. Reuth RG (1999) Goebbels J 1924-1945. Piper, München

14. Hanfstaengl E (1980) Zwischen weissem und braunem Haus. Piper, München

15. Hellpach W (1948) Wirken und Wirren. Lebenseriinerungen, eine Rechenschaft überWert und Glück, Schuld und Sturz meiner Generation. Band I und II. Christian Wegner, Hamburg

16. Hellpach W (1951) Tedeum. Laienbrevier einer Pantheologie. Christian Wegner Verlag, Hamburg

17. Hitler A (2016) Monologe. In: Hartmann C, Vordermayer T, Plöckinger O, Töppel R (Hrsg) Mein Kampf, eine kritische Edition, Bd. 1. Im Auftrag des Instituts für Zeitgeschichte, München, Berlin, S 286

18. Hoff P (2015) The Kraepelinian tradition. Dialogues Clin Neurosci 17:31-41

19. Hoppe JD (1997) Die Weiterbildungsordnung - von der Schilderordnung zum integralen Bestandteil der Bildung im Arztberuf. Dtsch Ärztebl 94:2483-2491

20. von Kemnitz M (1913) Der asthenische Infantilismus des Weibes in seinen Beziehungen zur Fortpflanzungstätigkeit und geistigen Betätigung. Arch Rassen Gesellschaftsbiol 42(10):41-66

21. von Kemnitz M (1914) Moderne Mediumforschung. Kritische Betrachtungen zu Dr. von Schrenck-Notzing's „Materialisationsphänomene". J F Lehmanns Verlag, München (Mit einem Nachwort von Dr. med. W.Gulat-Wellenburg)

22. von Kemnitz M (1917) Funktionelle Erkrankungen infolge von Kriegsbeschädigungen bei Offizieren. Neurol Cbl 36:230-233 
23. Koehne S (2013) Reassessing the "Holy Reich": leading Nazis' views on confession, community and Jewish materialism. J Contemp History 48:423-445

24. Kraepelin E (1915) Psychiatrie, ein Lehrbuch für Studierende und Ärzte. IV. Band. Klinische Psychiatrie III. Teil. 8. Barth, Leipzig

25. Krauss M (1997) „Man denke sich nur die junge Dame im Seziersaal ... vor der gänzlich entblössten männlichen Leiche", Sozialprofil und Berufsausübung weiblicher Ärzte zwischen Kaiserreich und Republik. In: Häntzschel $\mathrm{H}$, Bussmann H (Hrsg) Bedrohlich gescheit. CH Beck, München, S137-151

26. Krauss M (2005) „Die neue Zeit mit ihren neuen Forderungen verlangt auch eine neues Geschlecht ${ }^{\prime \prime}$ Die Ärztin Hope Bridges Adams Lehmann und ihre Forderungen an die Frau des 20. Jahrhunderts. In: Stahnisch F, Steger F (Hrsg) Medizin, Geschichte und Geschlecht. Körperhistorische Rekonstruktionen von Identitäten und Differenzen, Bd. 205. Steiner, Stuttgart, S 119-135

27. LudendorffE (1935) Der totale Krieg. Ludendorffs Verlag, München

28. Ludendorff M (2006) Der Seele Ursprung und Wesen. Zweiter Teil. Des Menschen Seele. Hohe Warte, Pähl

29. Ludendorff M (1936) Durch Forschen und Schicksal zum Sinn des Lebens. II. Teil von: Statt Heiligenschein oder Hexenzeichen mein Leben. Ludendorffs Verlag, München

30. Ludendorff M (1947) Kritik an dem Gutachten des Prof. Stertz. Staatsarchiv München, SpkA 1082 (Ludendorff)

31. Ludendorff M (1967) Freiheitskampf wider eine Welt von Feinden an der Seite des Feldherrn Ludendorff. V. Teil von: Statt Heiligenschein und Hexenzeichen mein Leben. Ludendorffs, München

32. LudendorffM, Ziersch W (1929) Alsich Ludendorffs Frau war. Drei Masken, München

33. Martini W (1949) Die Legende vom Hause Ludendorff. Inngau-Verlag Leonhard Lang, Rosenheim

34. Messias E (2014) Standing on the shoulders of Pinel, Freud, and Kraepelin. A historiometric inquiry into the histories of psychiatry. J Nerv Ment Dis 202:788-792

35. Möller HJ (1978) Psychoanalyse. Wilhelm Fink München

36. Niethammer L (1982) Die Mitläuferfabrik. Die Entnazifizierung am Beispiel Bayerns. S. Fischer, Berlin

37. Poewe K (2008) Scientific neo-paganism and the extreme right then and today: from Ludendorff's Gotterkenntnis to Sigrid Hunke's Europas Eigene religion. JContemp Religion 14:387-400

38. Polizeimeldebogen (1919) Kemnitz, von, Gustav. Stadtarchiv München, PMB IV 152, G153

39. Prokop J, Karenberg A (2020) Martha Ulrich und Toni Schmidt-Kraepelin, Pionierinnen der deutschen Nervenheilkunde. Nervenheilkunde 39:801-809

40. Radler R (1987) Ludendorff, Mathilde. Neue Dtsch Biogr 15:290-292

41. Richartz WE (1969) Tod den Ärtzten. Diogenes, Zürich

42. Schiller F (1793) Über Anmut und Würde. Neue Thalia. Leipzig, 2. Jahrgang, Heft 2

43. von Schrenck-Notzing Frhr A (1914) Materialisationsphaenomene. Ein Beitrag zur Erforschung der mediumistischen Teleplastie. Ernst Reinhardt München

44. von Schrenck-Notzing Frhr A (1932) Die Entwicklung des Okkultismus zur Parapsychologie in Deutschland. Mutze, Leipzig
45. Sommer A (2009) Tackling taboos-from psychopathia sexualis to the materialisation of dreams: Albert von Schrenck-Notzing (18962-1929). J Sci Explor 23:299-322

46. Spilker A (2009) Geschlechterverhältnisse und Zukunftsvorstellungen bei der Ärztin und völkischen Aktivistin Mathilde Ludendorff (1877-1966). Feministische Studien. De Gruyter, Stuttgart, S210-224

47. Spilker A (2013) Geschlecht, Religion, und völkischer Nationalismus. Die Ärztin und Antisemitin Mathilde von Kemnitz-Ludendorff (1877-1966). Campus, Frankfurt

48. Stertz G (1947) Gutachten, Spruchkammerverfahren gegen Frau Dr. Mathilde von Ludendorff. Staatsarchiv München, SpkA 1981 München

49. Töllner A (2013) „Klägliches Schauspiel der Versklavung einer Deutschen Seele durch eine Frau", Mathilde und Erich Ludendorff und das evangelische München. Theol Beih 7:193-215

50. von Unruh I (1950) Erklärung. Staatsarchiv München, SpKAK 1082 (Ludendorff)

51. Weber MM (2006) Feindliche Brüder im Geist der Wissenschaft? Zum 150. Geburtstag von Emi Kraepelin und Sigmund Freud. Psychotherapie 11:142-150
Hier steht eine Anzeige. Springer 